\title{
AVALIAÇÃO DE CURVAS DE MATURAÇÃO DE LARANJAS POR ANÁLISE DE AGRUPAMENTO'
}

DIRCEU MATTOS JUNIOR², ANGELA FUENTES GONZALES ${ }^{3}$, JORGINO POMPEU JUNIOR ${ }^{4}$ e CLÓVIS PARAZZI ${ }^{5}$

\begin{abstract}
RESUMO - Foram avaliadas curvas de maturação de frutos de 17 clones e cultivares de laranjas-doces pela análise de agrupamento. Determinou-se o ${ }^{\circ}$ Brix e a acidez das laranjas no período de agosto a dezembro de 1995, em Cordeirópolis, SP, obtendo-se as curvas que descrevem o comportamento das variáveis ao longo do tempo. Com base no ajustamento de equações polinomiais, foram calculadas estimativas médias para ${ }^{\circ}$ Brix e acidez aos 70, 75, 80, 85 e 90 dias após o início da coleta de frutos para análises. Com os dados padronizados, obteve-se o agrupamento pela média de grupos de pares não balanceados. Distinguiram-se quatro grupos de clones e cultivares de laranjas quanto à maturação. Um dos grupos, formado apenas pelo clone Navelência, apresentou $13,4^{\circ}$ Brix e acidez de $0,83 \%$, no início do período considerado, mostrando maturação precoce em relação aos outros clones e cultivares. A cultivar Pêra também formou um grupo isolado, com a razão ${ }^{\circ}$ Brix/acidez superior a 12,0, em meados de outubro. Para o grupo formado pelas laranjas Natal, Folha Murcha, Old Bud Line, Cutter, Valência, Lue Ging Gong e Tuxpan foi revelada a segunda quinzena de novembro como a época adequada para colheita, enquanto que, o agrupamento dos clones Frost, Whits, Olinda, Late, Stone, Chaffei, Werley e Berry atingiu a mesma relação de sólidos solúveis e acidez após 40 dias.
\end{abstract}

Termos para indexação: análise de qualidade, citros, laranja-doce, germoplasma

\section{EVALUATION OF DIFFERENT ORANGE MATURATION CURVES BY MEANS OF CLUSTER ANALYSIS}

\begin{abstract}
Fruit maturation of seventeen sweet orange clones and cultivars were evaluated by cluster analysis. Soluble solids ( ${ }^{\circ}$ Brix) and acidity were determined during the spring season 1995, in Cordeirópolis, SP, Brazil. Polynomial equations were determined between August and December and values for those variables were estimated $70,75,80,85$ and 90 days after the initial fruit sampling. The data set was standardized to perform cluster analysis regarding the Euclidean distance measure and the linkage rule as UPGMA type. Four clusters were selected in regard to fruit maturation stage. Navelência, the earliest clone, showed $13.4^{\circ}$ Brix and $0.83 \%$ acidity in the beginning of the considered period. The Pêra cultivar, another single clone cluster, showed ratio higher than 12.0 after middle October. The Natal, Folha Murcha, Old Bud Line, Cutter, Valência, Lue Ging Gong and Tuxpan orange clones and cultivars formed a third cluster, which reached harvesting maturity during middle November, whereas, Frost, Whits, Olinda, Late, Stone, Chaffei, Werley and Berry clones reached the same stage forty days later.
\end{abstract}

Index terms: orange juice, quality, citrus, germplasm.

${ }^{1}$ Aceito para publicação em 31 de maio de 1999

Apoio Fundação Instituto Agronômico.

${ }^{2}$ Eng. Agr., M.Sc., Centro de Citricultura Sylvio Moreira, Instituto Agronômico (IAC), Rod. Anhanguera, km 158, Caixa Postal 04 CEP 13490-970 Cordeirópolis, SP.

${ }^{3}$ Aluna do Curso de Engenharia Agronômica, Centro de Ciências Agrárias, UFSCar, Rod. Anhangüera, km 174, Caixa Postal 158, CEP 13600-970 Araras, SP. Bolsista do CNPq.

${ }^{4}$ Eng. Agr., Dr., Centro de Citricultura Sylvio Moreira, IAC. Bolsista do CNPq. E-mail: jorgino@centrodecitricultura.br

${ }^{5}$ Eng. Agr., Dr., Centro de Ciências Agrárias, UFSCar.

\section{INTRODUÇÃO}

A citricultura paulista se caracteriza pela pequena diversidade de cultivares copa. Com a finalidade de ampliar o número de cultivares a serem utilizadas no mercado de frutas frescas ou produção de suco, o Centro de Citricultura Sylvio Moreira, do IAC, vem introduzindo germoplasmas provenientes de outros estados e países. Atualmente, o banco de germoplasmas de citros (BAG-Citros) possui cer- 
ca de 630 acessos de laranjas-doces de potencial interesse à citricultura paulista.

A avaliação de curvas de maturação representa um dos primeiros passos na caracterização desse material genético, que pode ser utilizado como critério de seleção, no campo, daqueles materiais que atendam às características desejáveis.

Os teores de sólidos solúveis ( ${ }^{\circ}$ Brix), acidez do suco cítrico e a razão sólidos solúveis pela acidez têm sido utilizados para a avaliação da maturação dos citros (Chitarra \& Campos, 1981; Nogueira, 1987; Russo, 1987). Contudo, quando se trata de um grande número de acessos, a avaliação dos resultados é difícil, em função do grande volume de dados que são coletados ao longo do tempo. Assim, a análise de agrupamento, permite uma avaliação comparativa de todas as curvas de maturação estudadas, pela formação de grupos homogêneos através de uma medida de similaridade entre indivíduos em relação ao conjunto de variáveis.

Ao contrário do procedimento comum a testes estatísticos, a análise de agrupamento é utilizada quando não se tem uma hipótese a priori a ser testada, revelando-se uma importante fase exploratória da pesquisa e constituindo-se numa opção ao estudo de maturação dos citros.

Objetivou-se avaliar 13 clones de laranja Valência e as cultivares Folha Murcha, Natal, Pêra e Valência quanto à maturação dos frutos, utilizando a análise de agrupamento para as variáveis ${ }^{\circ}$ Brix e acidez, e a análise de regressão para estudo de grupos homogêneos em função do tempo.

\section{MATERIAL E MÉTODOS}

Foram avaliados os frutos de 13 clones de laranja Valência do Banco Ativo de Germoplasma (BAG)-citros, introduzidos no Centro de Citricultura em 1983, via Embrapa-Centro Nacional de Pesquisa de Mandioca e Fruticultura (CNPMF), Cruz das Almas, BA, e originalmente provenientes da Austrália, EUA e México. Os clones são identificados como: Berry, Chaffei, Cutter, Frost, Late, Lue Gin Gong, Navelência, Old Bud Line, Olinda, Stone, Tuxpan, Werley e Whits. Também foram avaliados os frutos das cultivares Folha Murcha, Natal, Valência e Pêra, candidatas ao banco de matrizes do Centro de Citricultura Sylvio Moreira (CCSM)/IAC. As plantas com cerca de oito anos de idade foram plantadas num Latossolo Vermelho-Escuro, original- mente álico, de textura argilosa, enxertadas sobre limoeiro 'Cravo', com exceção da 'Valência' (enxertada sobre tangerineira 'Cleópatra'). A análise química da camada superficial do solo mostrou $\mathrm{pH}\left(\mathrm{CaCl}_{2}\right)$ de 4,9 e teores altos para $\mathrm{P}$ resina $\left(30 \mathrm{mg} \mathrm{dm}^{-3}\right)$ e $\mathrm{K}\left(3,0 \mathrm{mmol}_{\mathrm{c}} \mathrm{dm}^{-3}\right)$, e médio para saturação por bases (51\%) (Grupo Paulista..., 1994).

O regime de chuvas para o período considerado foi marcado por meses secos, de agosto a setembro, cuja precipitação mensal foi inferior a $35 \mathrm{~mm}$; contudo, a partir da segunda quinzena de outubro foram registrados valores acumulados de $140 \mathrm{~mm}$ (Fig. 1). As temperaturas médias mensais máxima e mínima do ar não foram consideradas atípicas. A maior variação foi verificada na temperatura média mínima, de $14,8^{\circ} \mathrm{C}$ em agosto e $18,5^{\circ} \mathrm{C}$ em novembro (Fig. 1).

Cada acesso do BAG-citros, seja clone ou cultivar, é representado por três plantas localizadas lado a lado em três linhas de plantio, sendo cada conjunto de três plantas disposto no campo segundo uma seqüência para épocas de maturação e para espécies (Domingues \& Tulmann Neto, 1998). Foram avaliadas as três plantas que compõem cada acesso de clones e cultivares estudados no banco ativo de germoplasma. Coletou-se, de cada árvore, quatro frutos na porção mais externa da copa e em posições eqüidistantes e uma internamente à copa, numa altura média de $1,5 \mathrm{~m}$ do solo. Assim, a amostra para cada acesso avaliado foi composta por 15 frutos. A coleta de frutos foi feita em intervalos aproximadamente quinzenais, entre 29 de agosto e 6 de dezembro de 1995, tomando-se o cuidado de não misturar frutos de diferentes floradas, como no caso da 'Pêra'

As amostras foram analisadas em triplicata, determinando-se $0{ }^{\circ}$ Brix por leitura de refratômetro e a acidez por titulação com hidróxido de sódio $0,3125 \mathrm{~N}$, expressando-se

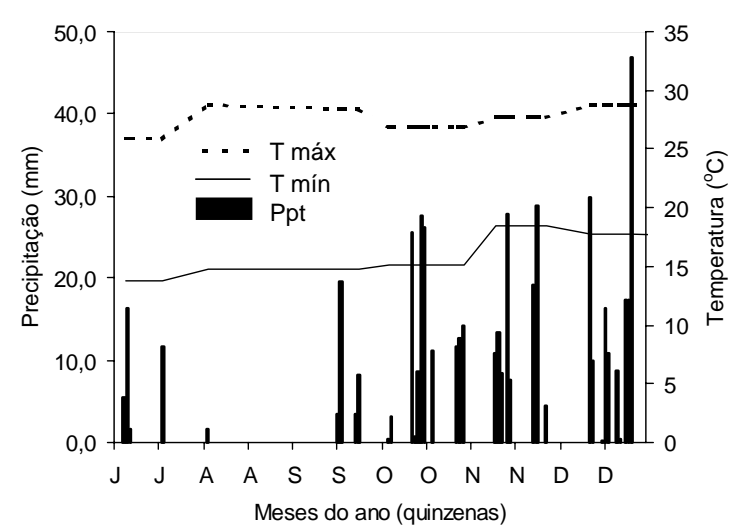

FIG. 1. Precipitação pluvial (Ppt) e temperaturas médias mensais máxima e mínima do ar. Cordeirópolis, SP, 1995. 
esse último em valor percentual para massa de ácido cítrico por volume de suco, segundo Reed et al. (1986). Calculouse a razão do ${ }^{\circ}$ Brix pela acidez.

Foi efetuado o ajustamento de uma equação polinomial global em todos os acessos, para as variáveis resposta ${ }^{\circ} \mathrm{Brix}$ e acidez como função do número de dias acumulados após o início da primeira coleta de frutos para análises (Ott, 1992). Definiu-se o valor zero para a primeira data de análise (29 de agosto) e, correspondentemente, os seguintes valores para as demais coletas: $22,38,59,76$ e 99 dias. O grau do modelo polinomial global que revelou melhor ajuste, julgado pelo coeficiente de determinação, foi adotado para o ajustamento de equações polinomiais individuais para cada um dos 17 acessos.

Estabeleceu-se, então, o intervalo de tempo em que o valor da acidez situou-se entre $0,8 \%$ e $1,2 \%$, condição em que, segundo Pannisi (1977), o fruto cítrico atinge qualidade de consumo durante o processo de maturação. Esse período foi dos 70 aos 90 dias após o início da coleta de frutos.

Para identificar esse intervalo como adequado à avaliação da maturação dos frutos, calculou-se a variação em torno das médias estimadas da razão ${ }^{\circ} \mathrm{Brix} /$ acidez para cada data de coleta, como $m \pm d p$, onde $m$ é a média estimada e $d p$ é o desvio-padrão da variável. Dispersões mais amplas sugerem maiores diferenças de maturação entre os clones e cultivares.

Com o auxílio das equações polinomiais para cada clone e cultivar, estimaram-se os valores médios para ${ }^{\circ}$ Brix e acidez aos 70, 75, 80, 85 e 90 dias após início da coleta de frutos. Como tais variáveis são não comensuráveis, efetuaram-se padronizações previamente à análise de agrupamento, segundo a expressão: $D_{\text {std }}=(D-m) / d p$, onde: $D_{s t d}$ é o dado padronizado; $D$ é o valor observado da variável resposta; $m$ e $d p$ são, respectivamente, a média e o desviopadrão das observações no período de avaliação

A análise de agrupamento foi realizada com auxílio do aplicativo "Statistica", versão 4.5 (Statsoft, 1994). Utilizouse o método de agrupamento da média aritmética não ponderada para grupos de pares ordenados (Sokal \& Michener, 1958), e a medida de distância das variáveis foi do tipo Euclidiana (Bussab et al., 1990; Manly, 1994).

O ponto de parada do processo de agrupamento sucessivo foi estabelecido arbitrariamente como aquele em que os grupos formados apresentam detalhamento apropriado da informação provida pelos dados, julgado pela experiência do pesquisador

Os dados de ${ }^{\circ}$ Brix e acidez para cada grupo formado após o corte do dendograma foram reagrupados para determinação de equações médias para curvas de maturação. Assim, obtiveram-se as curvas para ${ }^{\circ}$ Brix, acidez e razão ${ }^{\circ}$ Brix/acidez para cada grupo. No caso de agrupamentos representados por apenas um acesso, as equações foram as determinadas individualmente para aquele acesso.

\section{RESULTADOS E DISCUSSÃO}

As estimativas dos parâmetros das equações polinomiais ajustadas para os dados de ${ }^{\circ}$ Brix e acidez, globais e individuais para os 17 acessos, são apresentadas na Tabela 1. Os modelos quadrático para ${ }^{\circ}$ Brix e linear para acidez foram determinados pelo grau do modelo polinomial global, que revelou melhor ajuste, julgado pelo $\mathrm{r}^{2}$. Verificaram-se ajustes elevados para as equações globais, pois os coeficientes de determinação observados foram superiores a 0,97 ( $\mathrm{p}<0,01)$.

De acordo com Sinclair (1961), a concentração de ácido cítrico no fruto diminui durante o processo de maturação. Entretanto, com relação ao teor de sólidos solúveis, expresso em ${ }^{\circ}$ Brix, observouse uma tendência quadrática da variável, o que pode ser atribuído ao efeito da precipitação pluvial (Jones, 1961) que ocorreu com maior freqüência e intensidade após início do mês de outubro de 1995 (Fig. 1). Esse comportamento revelou uma média para a razão ${ }^{\circ}$ Brix/acidez de aproximadamente 12 para o período entre 70 e 90 dias após o início da análise (Fig. 2), que é considerado um valor mínimo desejável para colheita de frutos (Di Giorgi et al., 1990). Observa-se ainda, na Fig. 2, que a amplitude de variação (linhas verticais) em torno da média estimada para a razão ${ }^{\circ}$ Brix/acidez em relação aos 17 clones e cultivares avaliados é maior aos 76 dias após a coleta dos frutos, o que justifica a escolha dos dados estimados de ${ }^{\circ}$ Brix e acidez aos $70,75,80,85$ e 90 dias para compor a análise de agrupamento.

As equações polinomiais determinadas para cada acesso apresentaram também ajustes elevados $\left(\mathrm{r}^{2}>0,75 ; \mathrm{p}<0,01\right)$ (Tabela 1$)$, com poucas exceções. O ajustamento do modelo de ${ }^{\circ}$ Brix para a laranja Natal foi menor, tendo também sido observado, na acidez, ajustamentos menores em relação aos clones Stone e Navelência (Tabela 1). No caso deste último clone, o valor de $r^{2}$ foi muito pequeno, $o$ que poderia ser explicado pelo fato de que o resultado determinado no penúltimo intervalo de análise não seguiu a tendência, constante, da variável 
TABELA 1. Estimativas dos parâmetros das equações polinomiais ajustadas para exprimir a relação de ${ }^{\circ}$ Brix e acidez com o número de dias acumulados após início da coleta de frutos, e correspondentes coeficientes de determinação, global e individuais para os clones e cultivares de laranjas. Cordeirópolis, SP, 1995.

\begin{tabular}{|c|c|c|c|c|c|c|c|}
\hline \multirow[t]{2}{*}{ Copa } & \multicolumn{4}{|c|}{${ }^{\circ}$ Brix $^{1}$} & \multicolumn{3}{|c|}{ Acidez $^{2}$} \\
\hline & $\hat{a}$ & $\hat{b}$ & $\hat{\mathrm{c}}$ & $r^{2}$ & $\hat{a}$ & $\hat{b}$ & $r^{2}$ \\
\hline Global & $-0,0004$ & 0,0415 & 13,0040 & $0,99 * *$ & $-0,0072$ & 1,7377 & $0,97 * *$ \\
\hline 'Natal' & $-0,0004$ & 0,0319 & 12,4440 & $0,31^{\mathrm{NS}}$ & $-0,0087$ & 1,7841 & $0,88^{* *}$ \\
\hline 'F. Murcha' & $-0,0004$ & 0,0392 & 12,0650 & $0,87 * *$ & $-0,0097$ & 1,8372 & $0,83 * *$ \\
\hline 'Valência' & $-0,0003$ & 0,0277 & 13,2610 & $0,75^{* *}$ & $-0,0089$ & 1,7960 & $0,87 * *$ \\
\hline 'Pêra' & $-0,0005$ & 0,0316 & 14,2440 & $0,84 * *$ & $-0,0081$ & 1,5421 & $0,98 * *$ \\
\hline Cutter & $-0,0006$ & 0,0607 & 12,8370 & $0,99 * *$ & $-0,0056$ & 1,5979 & $0,94 * *$ \\
\hline Tuxpan & $-0,0005$ & 0,0450 & 11,7860 & $0,89 * *$ & $-0,0066$ & 1,5336 & $0,64 *$ \\
\hline Old Line & $-0,0003$ & 0,0315 & 12,0300 & $0,82 * *$ & $-0,0066$ & 1,6783 & $0,88 * *$ \\
\hline Navelência & $-0,0003$ & 0,0525 & 13,4170 & $0,85^{* *}$ & $-0,0002$ & 0,8250 & $0,03^{\mathrm{NS}}$ \\
\hline Frost & $-0,0005$ & 0,0477 & 12,2820 & $0,77 * *$ & $-0,0075$ & 2,0215 & $0,87 * *$ \\
\hline Olinda & $-0,0004$ & 0,0480 & 12,7010 & $0,85^{* *}$ & $-0,0057$ & 1,7263 & $0,94 * *$ \\
\hline Late & $-0,0006$ & 0,0377 & 13,8530 & $0,99 * *$ & $-0,0088$ & 1,9109 & $0,96^{* *}$ \\
\hline Lue & $-0,0005$ & 0,0440 & 13,4370 & $0,77 * *$ & $-0,0092$ & 1,7403 & $0,89 * *$ \\
\hline Stone & 0,0005 & 0,0491 & 13,5610 & $0,96 * *$ & $-0,0029$ & 1,6245 & $0,41^{\mathrm{NS}}$ \\
\hline Werley & $-0,0005$ & 0,0462 & 13,2320 & $0,96 * *$ & $-0,0064$ & 1,8168 & $0,91 * *$ \\
\hline Chaffei & $-0,0004$ & 0,0319 & 13,9450 & $0,88 * *$ & $-0,0094$ & 2,0456 & $0,97 * *$ \\
\hline Whits & $-0,0006$ & 0,0514 & 13,3230 & $0,92 * *$ & $-0,0086$ & 2,0663 & $0,90 * *$ \\
\hline Berry & $-0,0004$ & 0,0293 & 13,5920 & $0,84 * *$ & $-0,0088$ & 1,9939 & $0,96^{* *}$ \\
\hline
\end{tabular}

$1{ }^{\circ}$ Brix: $\hat{y}=\hat{a} x^{2}+\hat{b} x+\hat{c}$

${ }^{2} \operatorname{Acidez}(\%): \hat{y}=\hat{a} x+\hat{b}$

Ns, $*$ e $* *$ Não-significativo e significativo a $5 \%$ e $1 \%$ de probabilidade, respectivamente.

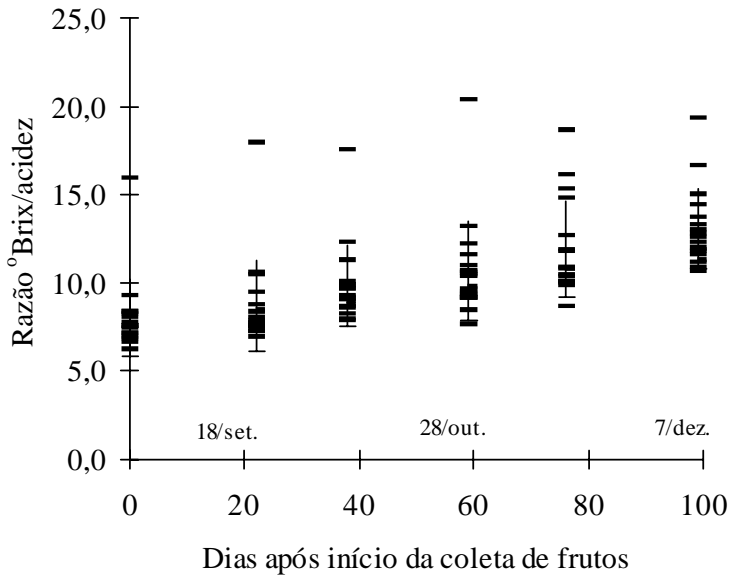

FIG. 2. Diagrama de dispersão das razões ${ }^{\circ}$ Brix/acidez calculadas nas seis coletas de frutos dos 17 clones e cultivares de laranjas. Linhas verticais indicam os intervalos de variação em torno das médias estimadas para cada data, calculados com base na equação $m \pm d p$, onde $m$ é a média estimada e $d p$ é o desvio-padrão da variável. (cerca de $0,82 \%$ ) verificada ao longo do período de análises dos frutos.

$\mathrm{O}$ dendograma de agrupamento mostrou quatro grupos distintos quando se utilizou a separatriz de seis unidades (Fig. 3). O primeiro grupo (G1) foi formado pelas laranjas Natal, Folha Murcha, Old Bud Line, Cutter, Valência, Lue Ging Gong e Tuxpan; o segundo grupo (G2) apresentou somente a cultivar Pêra; o terceiro (G3), os clones Frost, Whits, Olinda, Late, Stone, Chaffei, Werley e Berry; e por último, o grupo G4, com o clone Navelência.

As estimativas dos coeficientes das novas equações polinomiais ajustadas para cada um dos quatro grupos de acessos, para as variáveis ${ }^{\circ} \mathrm{Brix}$, acidez e da razão ${ }^{\circ}$ Brix pela acidez, estão na Tabela 2 e são representadas na Fig. 4.

Na Fig. 4a, verifica-se que o grupo G1 apresentou os menores valores médios de ${ }^{\circ}$ Brix ao longo de todo o período considerado, com valor médio do agrupamento de 13,1 aos 80 dias, que se pode considerar uma provável época de safra, enquanto a 
Navelência (G4) mostrou valor médio mais elevado para o referido agrupamento na mesma data, $15,7^{\circ}$ Brix. A cultivar Pêra $(\mathrm{G} 2)$ e o G3 diferiram quanto ao ${ }^{\circ}$ Brix no início das curvas de maturação, mas apresentaram valores praticamente iguais aos 80 dias após o início das avaliações. Essas diferenças são consideradas importantes numa situação em que a qualidade da fruta determinada por cultivares ou épocas adequadas de colheita representa ganhos econômicos diretos e indiretos para o negócio agrícola (Caixeta Filho, 1993).

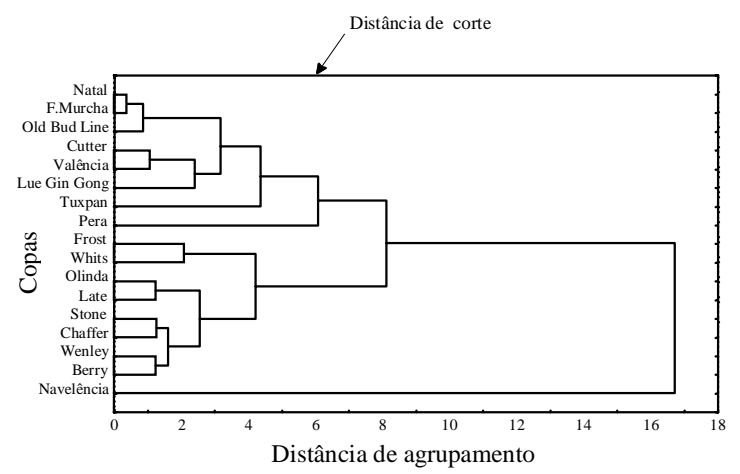

FIG. 3. Dendrograma de agrupamento para clones e cultivares de laranja obtido com valores das variáveis ${ }^{\circ}$ Brix e acidez estimados pelas equações polinomiais individuais aos 70,75 , 80,85 e 90 dias após início da coleta de frutos.
Com relação à acidez, verificou-se que os grupos G1 e G3 apresentaram teores médios mais elevados no início das avaliações e, embora decrescentes com o tempo, sempre foram superiores à 'Pêra' (G2) (Fig. 4b). Essa característica está relacionada ao fato de a cultivar Pêra ser considerada como de meia-estação quanto à maturação dos frutos na principal safra do ano, e as cultivares Valência, Natal e Folha Murcha, como tardias (Figueiredo, 1991). Foi notado comportamento distinto para a Navelência (G4), que não apresentou variações da acidez, embora tenha revelado acréscimos significativos de sólidos solúveis por cerca de três meses, o que possivelmente determina um período relativamente amplo para colheita (Fig. 4).

Rodriguez et al. (1973) avaliaram cultivares de laranja com maturação tardia, plantadas na Estação Experimental de Limeira, do IAC, em Cordeirópolis, SP. Os referidos autores avaliaram as cultivares Natal, Lue Ging Gong e Late, e num conjunto de dados sobre produção de frutos e da qualidade da fruta para colheita no mês de novembro, consideraram-nas promissoras para a citricultura. Os valores médios de ${ }^{\circ}$ Brix e acidez obtidos nesse trabalho foram, respectivamente, 10,7 e $0,81 \%$.

As curvas da razão ${ }^{\circ}$ Brix/acidez mostraram ocorrência de maturação mais precoce para a Navelência (G4), seguida da Pêra (G2), em relação às outras cultivares e clones de laranja Valência (Fig. 4c).

TABELA 2. Estimativas dos parâmetros das equações polinomiais ajustadas para exprimir a relação de ${ }^{\circ}$ Brix e acidez com o número de dias acumulados após início da coleta de frutos, e correspondentes coeficientes de determinação para os grupos de clones e cultivares de laranjas formados por agrupamento. Cordeirópolis, SP, 1995.

\begin{tabular}{|c|c|c|c|c|c|c|c|c|c|c|}
\hline \multirow[t]{2}{*}{ Grupo } & \multicolumn{4}{|c|}{${ }^{\circ} \mathrm{Brix}^{1}$} & \multicolumn{3}{|c|}{ Acidez $^{2}$} & \multicolumn{3}{|c|}{ Razão ${ }^{\circ}$ Brix/acidez } \\
\hline & $\hat{a}$ & $\hat{b}$ & $\hat{\mathrm{c}}$ & $\mathrm{r}^{2}$ & $\hat{a}$ & $\hat{\mathrm{b}}$ & $\mathrm{r}^{2}$ & $\hat{a}$ & $\hat{\mathrm{b}}$ & $\mathrm{r}^{2}$ \\
\hline G1 & $-0,0004$ & 0,0400 & 12,4160 & $0,96 * *$ & $-0,0079$ & 1,7096 & $0,94 * *$ & 0,0627 & 7,1910 & $0,97 * *$ \\
\hline $\mathrm{G} 2$ & $-0,0005$ & 0,0316 & 12,2400 & $0,84 * *$ & $-0,0081$ & 1,5421 & $0,98 * *$ & 0,0782 & 9,1340 & $0,98 * *$ \\
\hline G3 & $-0,0005$ & 0,0427 & 13,3110 & $0,97 * *$ & $-0,0074$ & 1,9007 & $0,96 * *$ & 0,0413 & 7,1238 & $0,98 * *$ \\
\hline G4 & $-0,0003$ & 0,0525 & 13,4170 & $0,85^{* *}$ & $-0,0002$ & 0,8250 & $0,03^{\mathrm{NS}}$ & 0,0331 & 16,6760 & $0,61 *$ \\
\hline
\end{tabular}

$1{ }^{\circ}$ Brix: $\hat{y}=a x^{2}+\hat{b} x+\hat{c}$

$2 \operatorname{Acidez}(\%): \hat{y}=\hat{a} x+\hat{b}$

${ }^{3}$ Razão ${ }^{\circ}$ Brix/acidez : $\hat{y}=\hat{a x}+\hat{b}$

Ns $*$ e ** Não-significativo e significativo a $5 \%$ e $1 \%$ de probabilidade, respectivamente. 

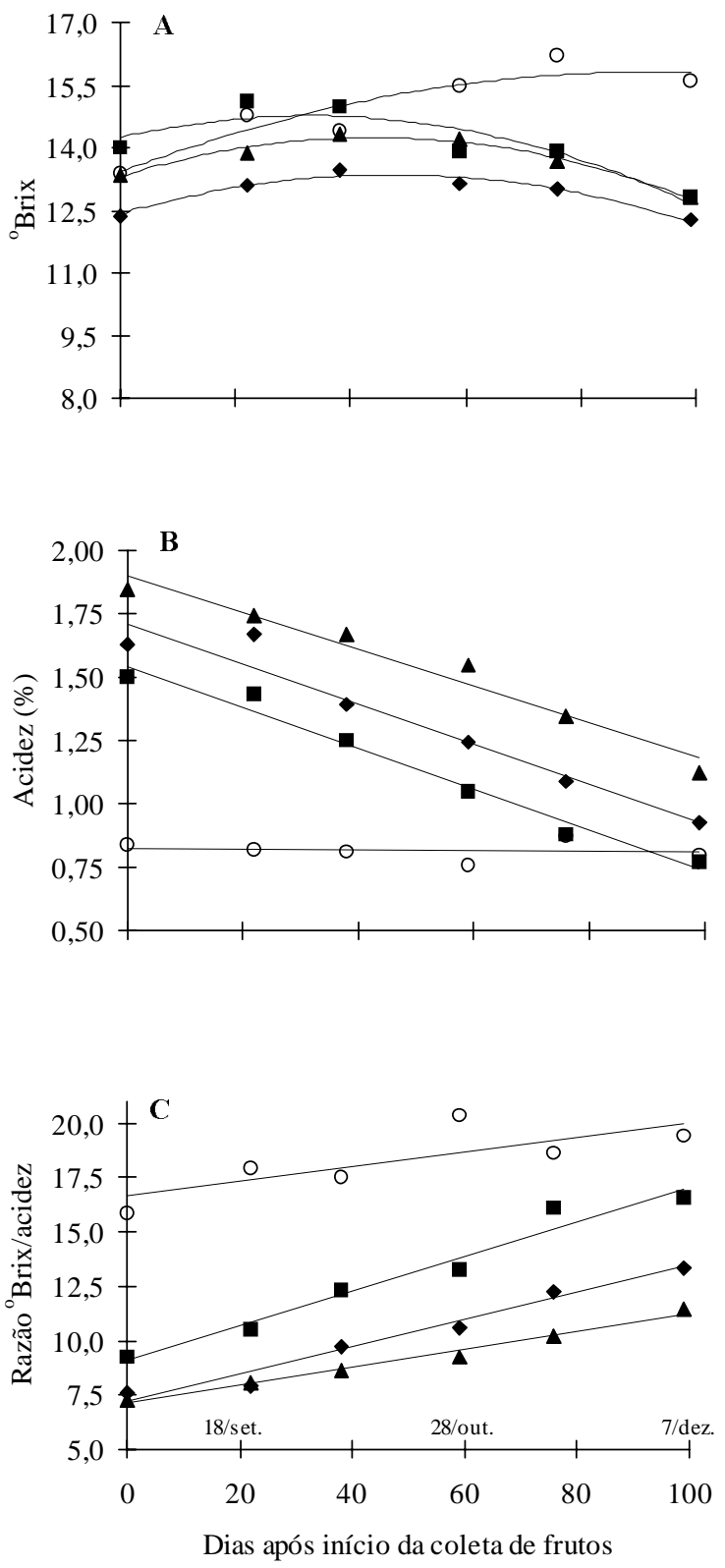

- Grupo 1 a Grupo 2 ^ Grupo 3 ○ Grupo 4

FIG. 4. Diagramas de dispersão das médias observadas de ${ }^{\circ}$ Brix, acidez e relação ${ }^{\circ}$ Brix/acidez nas seis datas de coletas de frutos, e correspondentes gráficos de linhas polinomiais ajustadas, para os quatro grupos de clones e cultivares de laranja determinados pela análise de agrupamento.
As equações ajustadas para exprimir a razão ${ }^{\circ}$ Brix/acidez média para os grupos G1 e G2 estimam que os dois grupos atingem a razão $12,0 \mathrm{com}$ uma diferença de cerca de 40 dias, ou seja, que o grupo G1 atinge essa razão aos 77 dias após o início da coleta de frutos, com acidez de $1,10 \% \mathrm{e}^{\circ} \mathrm{Brix}$ de 13,1 , enquanto o $\mathrm{G} 3$ atingiria razão ${ }^{\circ} \mathrm{Brix} /$ acidez semelhante, num período posterior a 99 dias após a coleta de frutos para análise (Fig. 4c).

É importante notar que os resultados discutidos neste trabalho são restritos às condições de solo e clima do local de estudo e ao ano em que os dados foram coletados. Trabalho como o de Chitarra \& Campos (1981) ilustra essa problemática com relação a diferentes regiões de estudo.

\section{CONCLUSÕES}

1. A análise de agrupamento é útil na avaliação de um grande número de curvas de maturação de laranjas.

2. Estabeleceram-se quatro grupos de clones e cultivares de laranjas com curvas de maturação distintas, formados pelas cultivares Pêra, Valência, Natal e Folha Murcha, tidas como referência.

3. A laranja Pêra, uma cultivar de meia-estação, forma um grupo isolado que apresenta razão ${ }^{\circ}$ Brix/acidez superior a 12,0 em meados de outubro.

4. Os clones Old Bud Line, Werley, Lue Ging Gong e Tuxpan são agrupados com as cultivares tardias Valência, Natal e Folha Murcha, enquanto o agrupamento dos clones Frost, Whits, Olinda, Late, Stone, Chaffei, Werley e Berry apresenta características de maturação mais tardia causada ainda pela alta acidez dos frutos.

5. A Navelência forma outro grupo, representado por um único clone, que mostra teores bastante baixos de acidez, praticamente constantes, e razão ${ }^{\circ}$ Brix/acidez elevada entre agosto e dezembro.

\section{REFERÊNCIAS}

BUSSAB, W.O.; MIAZAKI, E.S.; ANDRADE, D.F. Introdução à análise de agrupamentos. São Paulo: Associação Brasileira de Estatística, 1990. 104p. 
CAIXETA FILHO, J.C. Programação de colheita através do teor de sólidos solúveis. Laranja, Cordeirópolis, v.14, n.1, p.45-74, 1993.

CHITARRA, M.F.I.; CAMPOS, M.A.P. Caracterização de alguns frutos cítricos cultivados em Minas Gerais. I. Laranjas doces comuns (Citrus sinensis L. Osbeck) em fase de maturação. In: CONGRESSO BRASILEIRO DE FRUTICULTURA, 6., 1981, Recife. Anais. Recife: Sociedade Brasileira de Fruticultura, 1981. v.2, p.396-430

DI GIORGI, F.; IDE, B.Y.; DIB, K.; MARCHI, R.J.; TRIBONI, H.R.; WAGNER, R.L. Contribuição ao estudo do comportamento de algumas variedades de citros e suas implicações agroindustriais. Laranja, Cordeirópolis, v.11, n.2, p.567-612, 1990.

DOMINGUES, E.T.; TULMANN NETO, A. Bancos de germoplasma de citros e sua importância para o melhoramento. Laranja, Cordeirópolis, v.19, n.2, p.343364, 1998.

FIGUEIREDO, J.O. de. Variedades copa de valor comercial. In: RODRIGUEZ, O.; VIÉGAS, F.; POMPEU JÚNIOR, J.; AMARO. A.A. (Eds.). Citricultura brasileira. 2.ed. Campinas: Fundação Cargill, 1991. v. 2, p. $228-264$

GRUPO PAULISTA DE ADUBAÇÃO E CALAGEM PARA CITROS (Cordeirópolis, São Paulo). Recomendações de adubação e calagem para citros no Estado de São Paulo. Laranja, Cordeirópolis, v.15, 1994. 27p. Edição especial.

JONES, W.W. Environmental and cultural factors unfluencing the chemical composition and physical characters. In: SINCLAIR, W.B. (Ed.). The orange: its biochemistry and physiology. Berkeley: University of California, 1961 . p.25-55.

MANLY, B.F.J. Multivariate statistical methods: a primer. 2.ed. New York: Chapman and Hall, 1994 $215 \mathrm{p}$
NOGUEIRA, D.J.P. Evaluation of the internal chemical quality of citrus fruits. International Society of Citriculture . Proceedings, Piracicaba, v.2, p.520$522,1987$.

OTT, R.L. An introduction to statistical methods and data analysis. 4.ed. Belmont: Duxbury, 1992. 1051p.

PANNISI, L. Orange juice composition during maturation. In: CONGRESO MUNDIAL CITRICULTURA, 1., 1973, Murcia. [Anales]. Murcia: Instituto Nacional de Investigaciones Agrarias/Ministerio de Educacion y Ciencia, 1977. v.3, p.313-321.

REED, J.B.; HENDRIX JUNIOR, C.M.; HENDRIX, D.L. Quality control manual for citrus processing plants. Safety Habour: Intercit, 1986. v.1.

RODRIGUEZ, O.; SALIBE, A.A.; POMPEU JUNIOR, J.; FIGUEIREDO, J.O. de. Estudos de cultivares de laranjas de maturação tardia. In: CONGRESSO BRASILEIRO DE FRUTICULTURA, 1., 1971, Campinas. Anais. Campinas: Sociedade Brasileira de Fruticultura, 1973. v.1, p.267-276.

RUSSO, G. Ripening process of two orange cultivars: washington navel and navelina. International Society of Citriculture . Proceedings, Piracicaba, v.2, p.523$528,1987$.

SINCLAIR, W.B. Principal juice components. In: SINCLAIR, W.B. (Ed.). The orange: its biochemistry and physiology. Berkeley: University of California, 1961.p.131-160.

SOKAL, R.R.; MICHENER, C.O. A statistical method for evaluating systematic relationships. The University of Kansas Science Bulletin, Lawrence, v.38, n.22, p.1409-1438, 1958

STATSOFT (Tulsa, Estados Unidos). Statistica for Windows. In: — (Ed.). Statistics II. Tulsa, 1994. v. 3, p.30013911. 